\title{
Absorbing phase transitions in a non-conserving sandpile model
}

\author{
Marvin Göbel \& Claudius Gros \\ Institute for Theoretical Physics, Goethe University Frankfurt, Frankfurt a.M., \\ Germany \\ E-mail: gros07 [myPlace] @itp.uni-frankfurt.de
}

March 2019

\begin{abstract}
We introduce and study a non-conserving sandpile model, the autonomously adapting sandpile (AAS) model, for which a site topples whenever it has two or more grains, distributing three or two grains randomly on its neighboring sites, respectively with probability $p$ and $(1-p)$. The toppling process is independent of the actual number of grains $z_{i}$ of the toppling site, as long as $z_{i} \geq 2$. For a periodic lattice the model evolves into an inactive state for small $p$, with the number of active sites becoming stationary for larger values of $p$. In one and two dimensions we find that the absorbing phase transition occurs for $p_{c} \approx 0.717$ and $p_{c} \approx 0.275$.

The symmetry of bipartite lattices allows states in which all active sites are located alternatingly on one of the two sublattices, A and B, respectively for even and odd times. We show that the AB-sublattice symmetry is spontaneously broken for the AAS model, an observation that holds also for the Manna model. One finds that a metastable AB-symmetry conserving state is transiently observable and that it has the potential to influence the width of the scaling regime, in particular in two dimensions.

The AAS model mimics the behavior of integrate-and-fire neurons which propagate activity independently of the input received, as long as the threshold is crossed. Abstracting from regular lattices, one can identify sites with neurons and consider quenched networks of neurons connected to a fixed number $G$ of other neurons, with $G$ being drawn from a suitable distribution. The neuronal activity is then propagated to $G$ other neurons. The AAS model is hence well suited for theoretical studies of nearly critical brain dynamics. We also point out that the waiting-time distribution allows an avalanche-free experimental access to criticality.
\end{abstract}

Keywords: absorbing phase transition, non-conserving sandpile model, neuronal sandpile model, brain criticality

\section{Introduction}

Dynamical systems with self-propagating activities are said to undergo an absorbing phase transition when changes in a control parameter lead to a transition from an active to an inactive, an absorbing state [1], or vice versa. Examples are contact processes [2,3], 
the conserved lattice gas [4], and sandpile dynamics on lattices with periodic boundary conditions [5].

Absorbing phase transitions are potentially important for the quasi-stationary neuronal activity of the brain, which is continuously propagated on the level of individual neurons. Indications of power-law avalanches [6, 7] have been interpreted in this context as signs of self-organized criticality [8]. Power laws may arise however also from branching processes that are tuned homeostatically to criticality [9], namely when the average activity is self regulated, and when observations sample dynamical states based on the size of their attractors $[10,11]$, as it is the case for vertex routing models $[12]$.

Resolving the questions whether the brain is close to a self-organized critical state [13], as occurring in sandpile models with open boundary conditions [14], is plagued by several issues. On a technical level it has been shown that sub-sampling needs to be taken into account when interpreting experiments [15]. Conceptually there is in addition the dichotomy between model and brain with respect to conservation laws. Sandpile models with open boundary conditions are self-organized critical only when locally conserving $[16,17]$, at least on the average [18], a property not shared by real-world neuronal cells.

Absorbing phase transitions in sandpile models with periodic boundary conditions have been considered hitherto to depend on toppling processes that conserve energy locally [19], or that allow for a fine tuning between dissipation and energy uptake [20]. Here we propose a new model, the autonomously adapting sandpile (AAS) model, for which the toppling events are generically non-conserving. Nevertheless, a transition between absorbing states and phases with self-regulated activity is observed. We present a first assessment of the AAS model, with a focus on several basic issues, such as the spontaneous breaking of the AB-sublattice symmetry and the presence of metastable states. Our results indicate that the AAS model is likely to fall into the universality class of directed percolation.

\subsection{The autonomously adapting sandpile model}

The toppling dynamics of the AAS model is a straightforward modification of the respective Manna model rules. A site with two or more grains topples, at which point either two or three grains are added randomly onto its neighboring sites, as illustrated in Fig. 1. The key difference to the Manna model is that the number of grains added to the neighboring sites is independent of the number $z_{i}$ of grains on the toppling site before toppling. Instead, three grains are distributed with probability $p$ and two grains with probability $1-p$. After toppling the formerly active site has zero grains associated with it. As shown in Fig. 2, one observes an absorbing phase transition, which occurs at $p_{c}=0.27523(1)$ in two dimensions and at $p_{c}=0.71692(2)$ in one dimension.

The mechanism leading to a self-organized activity level in the AAS model is closely related to the homeostatic adaption regulating the firing rate of real-world neurons. A site is active when the number of grains received is larger than a certain threshold, which 


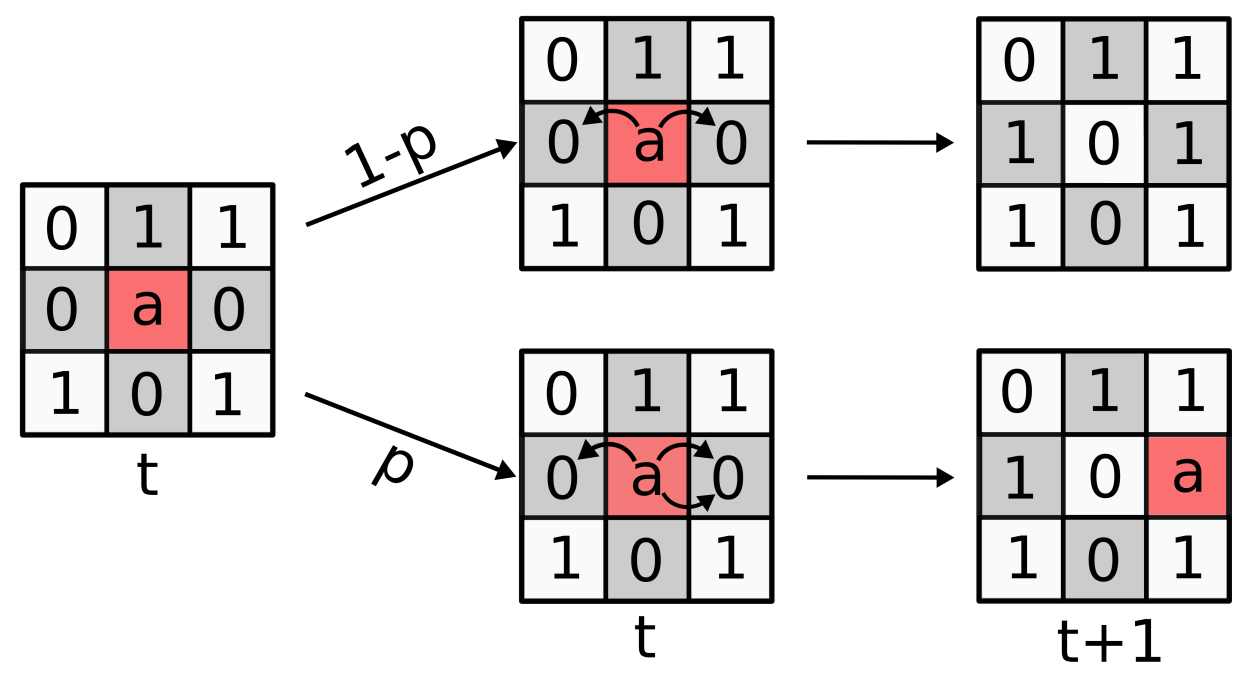

Figure 1. Illustration of the AAS toppling rules. Active sites (red) become empty after a toppling event, which consists to adding three or two grains randomly to neighboring sites, respectively with probabilities $p$ and $(1-p)$. Note that the number of grains added is independent of the occupation number $z_{i}$ of the toppling site, as long as $z_{i} \geq 2$. Sites may receive more than one grain.

is unity in the here considered case. The number of grains and with it the activity is down-regulated when $z_{i}$ is large, as no more than three grains can be distributed. The activity is on the other hand up-regulated when $z_{i}$ is small, in our case when $z_{i}=2$, as there is a finite probability $p$ to distribute more grains, namely three.

Within the AAS model discussed here, either two or three grains are distributed. Generalizing this rule one can define a distribution $p(G)$, such that $G>0$ grains are distributed during a toppling event with probability $p(G)$. Instead of a regular lattice one can consider in addition a network of sites having a coordination number, the average number of edges [19], that may be either small or large. We believe that the resulting model, the net-AAS, would capture key features of interconnected integrate-and-fire neurons and that it would be interesting to use the net-AAS model for theoretical studies of critical neuronal activity.

Of experimental interest are furthermore methods that allow to gauge the distance to criticality without the need to define avalanches. We point out that the distribution of waiting times between two topplings events is critical and easily measurable, as it corresponds to the time between subsequent neuronal spikes. For the AAS model we confirm that the mean waiting time scales inversely to the number of active sites [21], viz to the order parameter.

\section{Stochastic breaking of the AB-sublattice symmetry}

As bipartite lattices, one-dimensional chains and the square lattice decompose into two sublattices, A and B. Active sites on the A sublattice distribute grains exclusively 


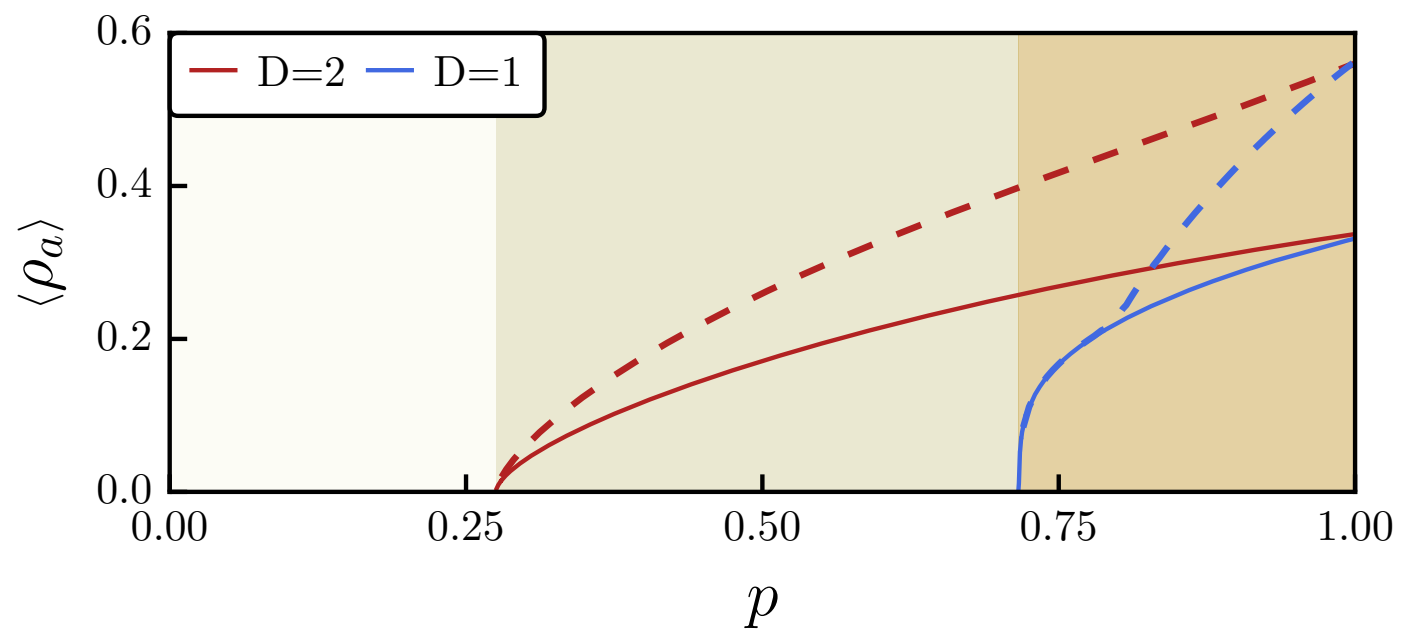

Figure 2. The density $\rho_{a}$ of active sites, as a function of the probability $p$ that a toppling event distributes three and not two grains, as illustrated in Fig. 1. For $L \times L$ square lattices with $L=128-512$ (red lines), and for a one-dimensional system with $10^{4}-10^{5}$ sites (blue lines). Synchronous updating has been used, which allows for metastable solution conserving the AB-sublattice symmetry (dashed lines), as discussed in Sect. 2. After prolonged times the system settles into a stable ABsublattice symmetry breaking solution (full lines). Close to the absorbing transition the metastable solution is not well defined, as evident is particular in one dimension. The transitions from an absorbing to an active phase occur at $p_{c} \approx 0.27453(1)$ and $p_{c} \approx 0.71692(2)$ in two and one dimensions.

to the B sublattice, and vice versa. The system may hence settle into a sequence of configurations for which all active sites are located alternatively on the $\mathrm{A}$ and the B sublattice. Such a sequence would imply that the AB-sublattice symmetry is dynamically broken. Whether this is possible depends however on the updating rule.

- Synchronous updating consists of saving the current configuration in a first step, with the subsequent toppling of the active sites occurring simultaneously [1].

- For sequential asynchronous updating one goes randomly through the list of active sites. Once processed, a new list of active sites is compiled [22].

- Random asynchronous updating implies in contrast that active sites are toppled randomly one by one. The configuration is updated after every toppling event [23].

It is clear that random asynchronous updating does not allow the AB-sublattice symmetry to be broken dynamically. For synchronous and sequential asynchronous dynamics one observes however, as shown in Fig. 3, that the AB-sublattice symmetry is spontaneously broken for the AAS and the Manna model. These results indicate that the AB-sublattice symmetry may be generically broken for sandpile models with nearest-neighbor dynamics on bipartite lattices.

We denote with $\rho_{a}^{A B}(t)$ the density of active sites on the A sublattice, when $t$ is even, and, alternatingly, the density of active sites on the B sublattice for odd times $t$. An equivalent definition holds for $\rho_{a}^{B A}(t)$. Starting from random initial conditions, 

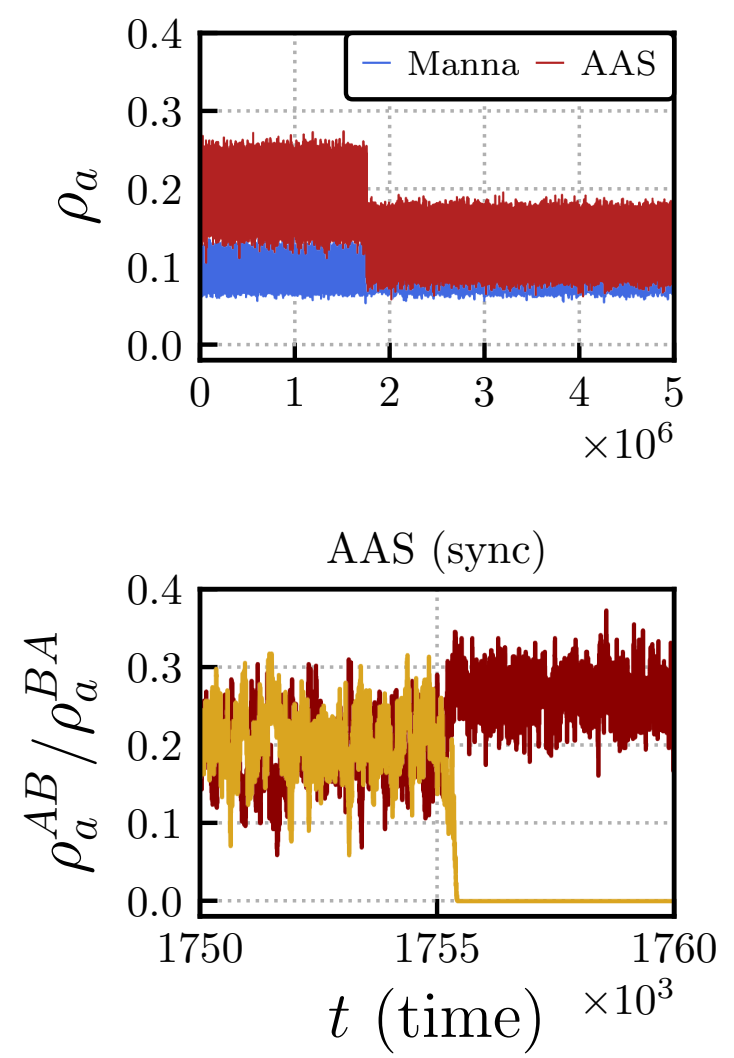
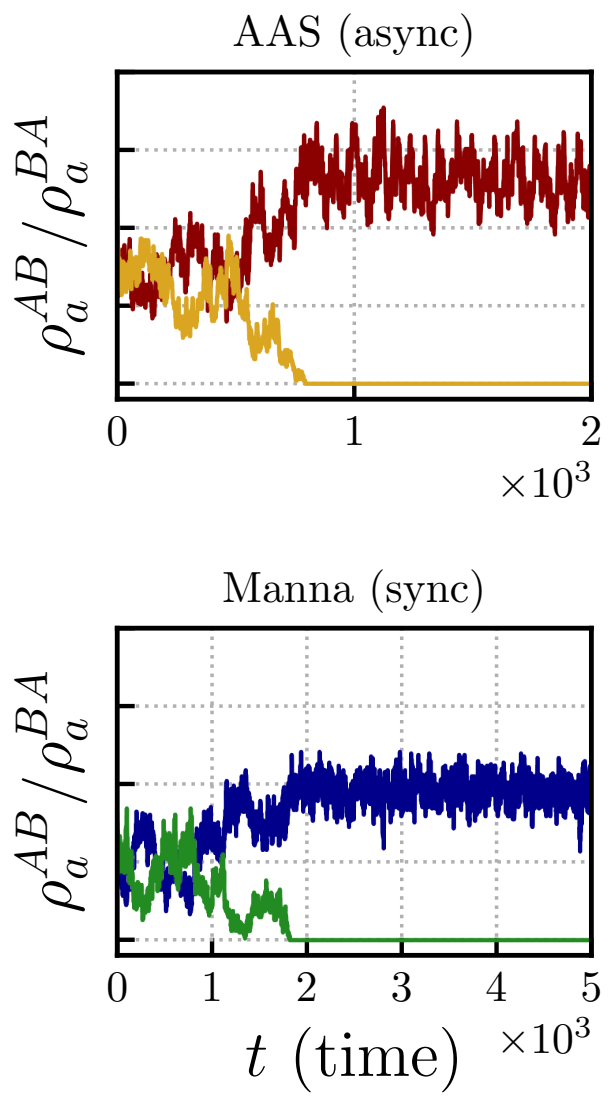

Figure 3. Timelines illustrating the spontaneous breaking of the AB-sublattice symmetry on a $32 \times 32$ square lattice, for the AAS model with $p=0.4$ (red and yellow curves) and for the Manna model with a grain density of 0.74 (blue and green curves). Shown are results for synchronous (sync) and sequential asynchronous (async) updating. Upper Left: The overall density of active sites, which remains finite, as both systems are in the active phase. Evident is a transition, which occurs after about $1.76 \cdot 10^{6}$ steps for the AAS model. At this scale the Manna model is already in a symmetry broken state. Upper Right: A close-up of the densities of active sites, $\rho_{a}^{\mathrm{AB}}$ and $\rho_{a}^{\mathrm{BA}}$, which alternate between the two sublattices for even/odd times. AAS model with asynchronous updating. Lower Left: $\rho_{a}^{\mathrm{AB} / \mathrm{BA}}$ for the AAS model with synchronous updating. Lower Right: $\rho_{a}^{\mathrm{AB} / \mathrm{BA}}$ for the Manna model with synchronous updating.

for which $\rho_{a}^{A B}(t)$ and $\rho_{a}^{B A}(t)$ are of comparable magnitude, one observes that $\rho_{a}^{A B / B A}(t)$ fluctuate until one of the sublattices is devoid of active sites, compare Fig. 3. At this point the AB-sublattice symmetry is fully broken.

Empirically we find that the time $t_{0}$ needed for breaking the AB-sublattice symmetry increases with system size. We did however restrain from attempting to determine the functional dependency of $t_{0}$ on the system size and on $p-p_{c}$, noting that $t_{0}$ tends to vary strongly from one simulation to another. We could hence not determine, at this point, whether the process leading to the breaking of the AB-sublattice symmetry shows finite-size and/or critical scaling. It is presently also not clear why $t_{0}$ differs by several orders of magnitude between the Manna and the AAS model, and/or for 

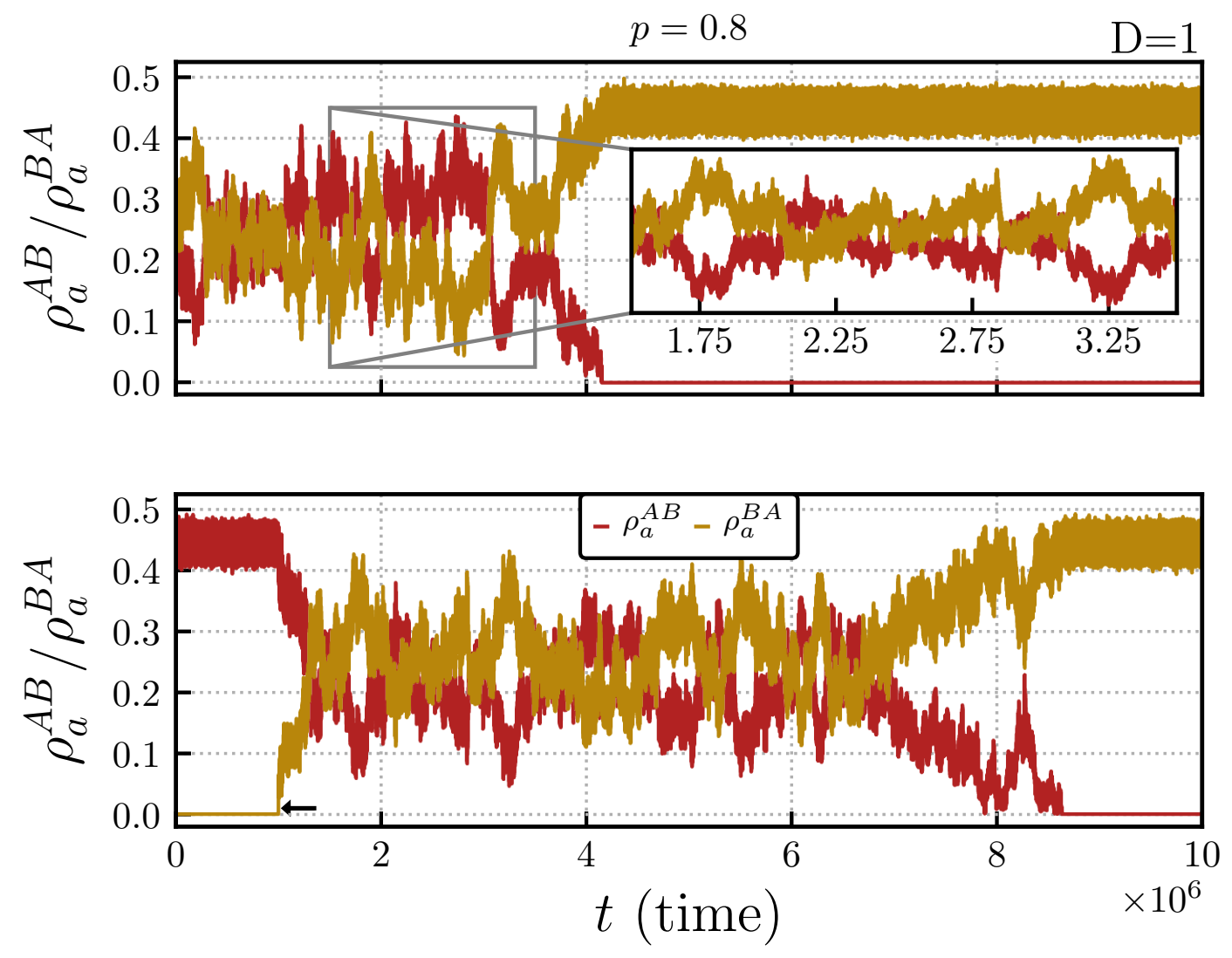

Figure 4. For $p=0.8$ the time series of the densities of active sites $\rho_{a}^{\mathrm{AB}} / \rho_{a}^{\mathrm{BA}}$ located alternating for even and odd times on the $\mathrm{A}$ and $\mathrm{B}$ sublattice in the one-dimensional AAS model with $L=10^{4}$. Below $p \approx 0.785$ the AB-conserving and broken states are nearly indistinguishable, compare Fig. 2. Upper panel: The symmetry is broken spontaneously after about $4 \cdot 10^{6}$ time steps. The symmetric state is characterized by visible drifts in $\rho_{a}^{\mathrm{AB}} / \rho_{a}^{\mathrm{BA}}$ whereas only small drifts can be observed in the asymmetric state. Lower panel: The system was initialized with a broken AB-symmetry. At $t=10^{6}$ the grains of $5 \%$ of the active sites were shifted by one lattice spacing in a random direction, leaving the total number of grains and active sites conserved. This procedure generates activity on the formerly inactive sublattice, as indicated by the horizontal arrow. For short times the symmetric metastable state is restored until the sublattice symmetry is broken anew by stochastic fluctuations.

synchronous vs. sequential asynchronous updating. This disparity indicates in any case that the AB-symmetry is broken via a stochastic process and not by a driving molecular field, viz already on a molecular-field level, a supposition that is in agreement with the mean-field results presented in Sect. 4.1.

\subsection{Metastable state}

The long timescales needed to break the AB-sublattice symmetry for synchronous update rules allow to study the metastable sublattice symmetric state. In Fig. 4 we present the evolution for the one-dimensional case, which indicates that stochastic 

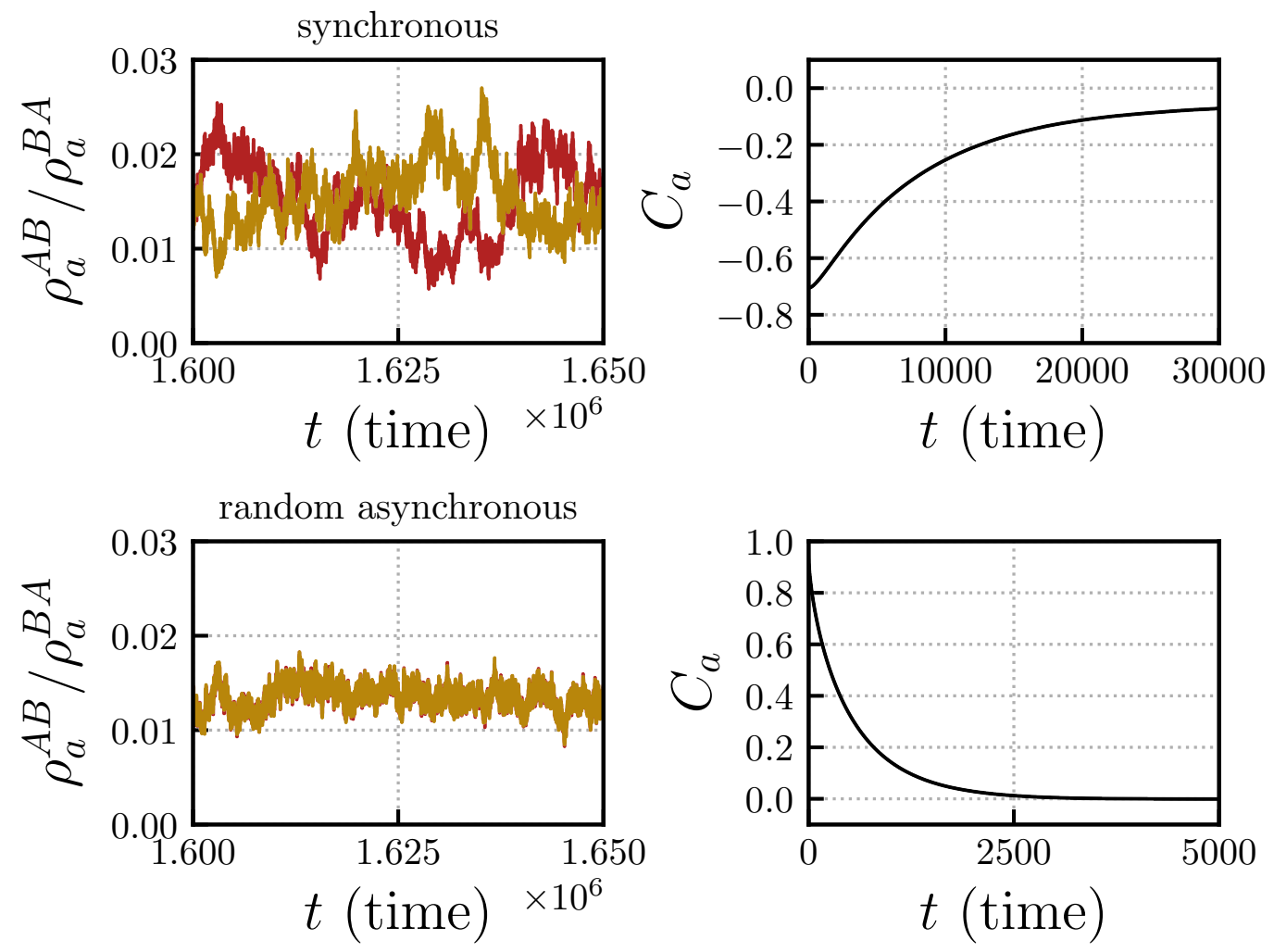

Figure 5. The timeline of the number of active sites located alternatingly on the $\mathrm{A}$ and $\mathrm{B}$ sublattice, $\rho_{a}^{\mathrm{AB}}$ and $\rho_{a}^{\mathrm{BA}}$. For a $512 \times 512$ square lattice with synchronous updating (upper panels) and with random asynchronous updating (lower panel). The AB-sublattice symmetry is not broken in the latter case. Here $p-p_{c}=4 \cdot 10^{-3}$. Also shown are the respective cross-correlation functions $C_{a}$, as defined by (1). Note that the sublattices are highly anti-correlated for the metastable state realized for synchronous updating.

tipping may cause the instability of the AB-symmetric state. For large densities of active sites the instability cannot be observed, presumably due to diverging time scales.

We note, as shown in Fig. 2, that the densities of active sites of the two states, ABsublattice conserving and not conserving, approach each other for $p \rightarrow p_{c}$. In 1D the two densities become virtually indistinguishable below $p \approx 0.785$, viz substantially above $p_{c}$. The stability time of the metastable state is however reduced when the densities of active sites of the two states become similar.

Also included in Fig. 4 is a protocol studying the short-term stability of the symmetry-broken state. Inducing a small number of active sites on the sublattice that was previously devoid of active sites leads to a short-term runaway growth, until the fluctuating sublattice-symmetric metastable state is reached. This implies that the asymmetric state is unstable on short time scales, but absorbing for long times, which is possibly a quite unique situation.

A symmetry can be broken stochastically only if the broken state is dynamically stable, viz absorbing. A stochastically broken symmetry could hence influence the 
universality class [24]. It seems however, that the exponents found numerically for models that are prone to break the AB-sublattice symmetry are independent of the update rules used $[25,26]$. We did not investigate these issues further, concentrating in this study for the most part on the ASS model with synchronous updating.

\subsection{Correlations in the metastable state}

In order to obtain an improved understanding of the metastable state we show in Fig. 5 a typical timeline for a $512 \times 512$ two-dimensional system close to the transition point, specifically for $p-p_{c}=4 \cdot 10^{-3}$. The densities of active sites on alternating sublattices, $\rho_{a}^{\mathrm{AB}}$ and $\rho_{a}^{\mathrm{BA}}$, are manifestly anticorrelated. For a quantitative measure we evaluated the time-dependent cross-correlation function

$$
C_{a}(t)=\frac{\left\langle\left(\rho_{a}^{A B}(t)-\left\langle\rho_{a}^{A B}\right\rangle\right)\left(\rho_{a}^{B A}(0)-\left\langle\rho_{a}^{B A}\right\rangle\right)\right\rangle}{\sigma^{A B} \cdot \sigma^{B A}},
$$

where

$$
\sigma^{A B}=\sqrt{\left\langle\left(\rho_{a}^{A B}-\left\langle\rho_{a}^{A B}\right\rangle\right)^{2}\right\rangle}, \quad \sigma^{B A}=\sqrt{\left\langle\left(\rho_{a}^{B A}-\left\langle\rho_{a}^{B A}\right\rangle\right)^{2}\right\rangle} .
$$

One observes that $C_{a}$ is always negative, starting out at about -0.7 , which implies a substantial anticorrelation of the two sublattices.

Included in Fig. 5 are the results for a simulation using random asynchronous updating, for which toppling events are treated individually, as explained further above. For random asynchronous dynamics the sublattice symmetry can not be broken. Interestingly, the cross-correlation function $C_{a}$ is in this case always positive, decaying furthermore substantially faster than for synchronous updating. The respective times to decay to half-heights are about 6000 and 300, respectively for synchronous and random updating. Note that the time unit is given in the synchronous by a sweep and in the asynchronous by $N_{a}$ topping events, where $N_{a}$ is the current number of active sites, which implies that the two time units are comparable. It would be interesting, but beyond the scope of the present investigation, to study the metastable state in more detail, as well as the properties of systems with random asynchronous updating.

\section{Critical properties}

Numerical simulations of the AAS model are somewhat demanding, as the number of grains fluctuates in addition to the number of active sites. We performed simulations in one dimension and for $L \times L$ square lattices, using synchronous updating, which defines a time step as a sweep over all active sites. Typically we reserved $5 \cdot 10^{7}$ simulation steps for equilibration, with the actual measurements taking about $10^{8}$ time steps, which reflects the procedure used in comparable studies $[20,28]$. We checked that the initial grain density is irrelevant, viz that the steady-state density $g=g(p)$ is uniquely a function of $p$. No differences with respect to synchronous updating could be found when performing somewhat less extensive simulations with sequential and random asynchronous updating 
rules, as defined in Sect. 2. For the correlation length, we found that the numerical efforts required to obtain reliable results are exceedingly demanding. This topic was hence left to future studies.

For synchronous updating it may take a substantial time for the AB-sublattice symmetry to be broken stochastically, as described in Sect. 2. In order to make sure that we work in the symmetry broken state we used symmetry breaking initial conditions, with the overall density of grains being one. Grains were added one by one to a site either if the site was on the A sublattice, or if it was empty.

\subsection{Scaling exponents}

For the densities $\left\langle\rho_{a}\right\rangle$ and $\langle g\rangle$ of active sites and respectively of grains, the scaling exponents are defined as

$$
\left\langle\rho_{a}\right\rangle \sim\left(p-p_{c}\right)^{\beta}, \quad\langle g\rangle-g_{c} \sim\left(p-p_{c}\right)^{\tilde{\beta}},
$$

where we have used with $\beta$ and $\tilde{\beta}$ a notation that relates the exponents of the grain density to the ones characterizing the scaling of the density of active sites. The time averaged fluctuations of the density of active sites and grains,

$$
\Delta \rho_{a}=L^{2}\left\langle\left(\rho_{a}-\left\langle\rho_{a}\right\rangle\right)^{2}\right\rangle, \quad \Delta g=L^{2}\left\langle(g-\langle g\rangle)^{2}\right\rangle,
$$

come with respective exponents,

$$
\Delta \rho_{a} \sim\left(p-p_{c}\right)^{-\gamma^{\prime}}, \quad \Delta g \sim\left(p-p_{c}\right)^{-\tilde{\gamma}^{\prime}} .
$$

We also evaluated the mean time $\left\langle\tau_{\text {wait }}\right\rangle$ between two toppling events on the same site, the waiting time,

$$
\left\langle\tau_{\text {wait }}\right\rangle=1 /\left\langle\rho_{a}\right\rangle \sim\left(p-p_{c}\right)^{-\beta}
$$

where we have included that the waiting time is inversely proportional to the density of active sites, compare (4). Within the neural network interpretation the mean waiting time corresponds to the average interspike interval, which is experimentally accessible.

Table 1. One-dimensional critical exponents. Note that $\tilde{\beta}, \tilde{\gamma}^{\prime}$ and $\tilde{\sigma}$ are defined only for the autonomously adapting sandpile model (AAS), see (3) and (5) and (7). Manna and directed percolation (DP) exponents are from [27]. The exponent $\epsilon_{\|} / \nu_{\|}$for the waiting time distribution is defined by (9).

\begin{tabular}{llll}
$1 \mathrm{D}$ & $\mathrm{AAS}$ & Manna & $\mathrm{DP}$ \\
\hline$\beta$ & $0.278(5)$ & $0.382(19)$ & $0.276486(8)$ \\
$\tilde{\beta}$ & $0.293(14)$ & - & - \\
$\gamma^{\prime}$ & $0.54(3)$ & $0.55(4)$ & $0.543882(16)$ \\
$\tilde{\gamma}^{\prime}$ & $0.52(3)$ & - & - \\
$\sigma$ & $2.40(8)$ & $2.71(4)$ & $2.554216(13)$ \\
$\tilde{\sigma}$ & $2.46(9)$ & - & - \\
\hline$\epsilon_{\|} / \nu_{\|}$ & $1.86(9)$ & - & $1.840536(5)$
\end{tabular}



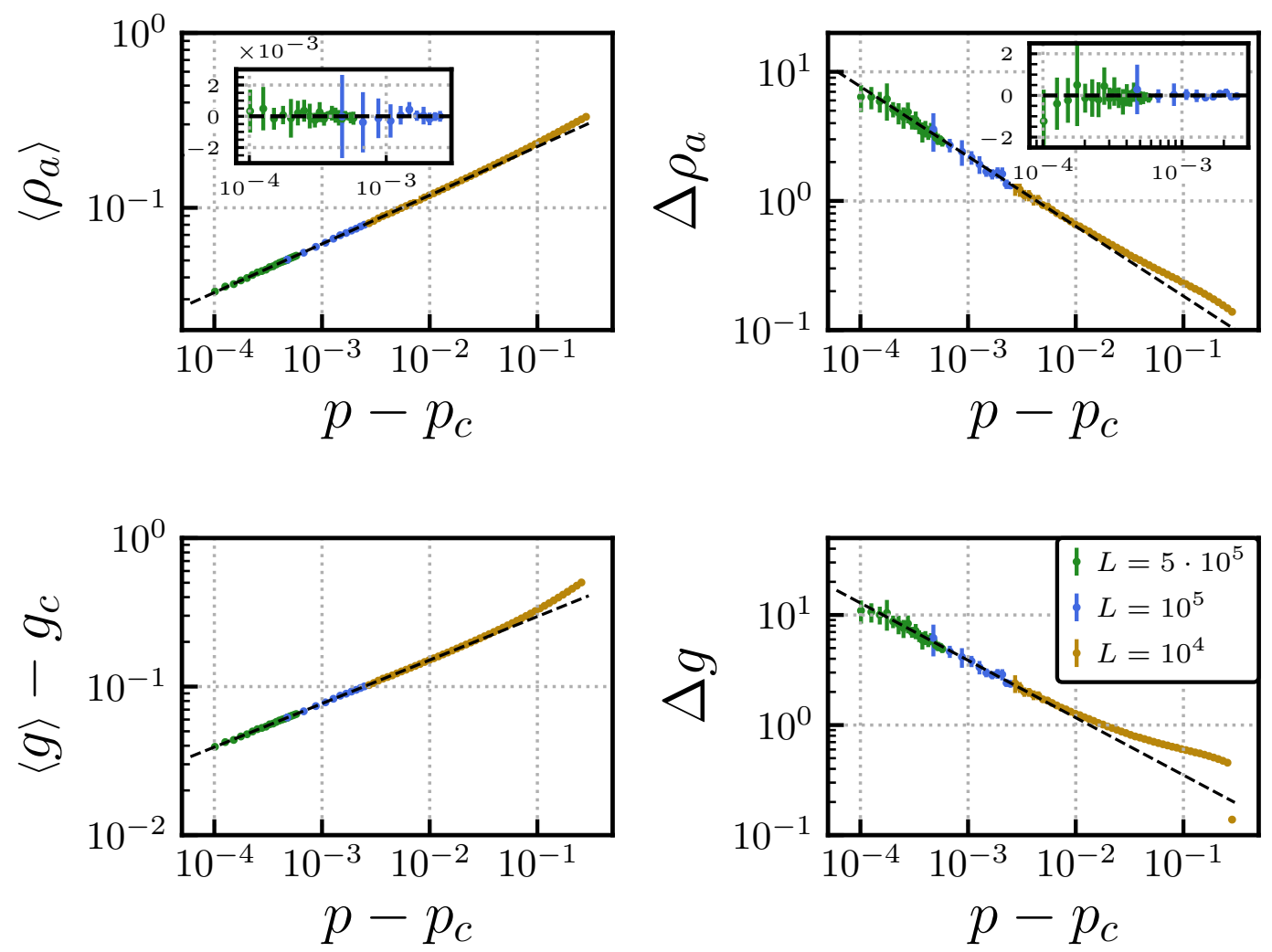

Figure 6. For one-dimensional chains of length $L$, the moments of the density of active sites and grains in the AB-symmetry broken state. In the insets for $\left\langle\rho_{a}\right\rangle$ and $\Delta \rho_{a}$ the error relative to the respective power-law fit is shown in units of $10^{-3}$ and $10^{0}$. The fits for $\left\langle\rho_{a}\right\rangle$ and $\langle g\rangle-g_{c}$ where performed simultaneously, assuming a common critical probability to distribute three and not two sites, $p_{c}=0.71692(2)$, upon toppling. The resulting critical density of grains is $g_{c}=0.733(6)$. Data for up to $p=1$ is shown. Upper panels: The density and the fluctuations of active sites as a function of $p-p_{c}$ (colored symbols), in a log-log representation, together with the optimal fit (dashed line). The exponents are $\beta=0.278(5)$ and $\gamma^{\prime}=0.54(3)$. Lower panels: As for the upper panels, but for the mean grain density $\langle g\rangle$. The exponents are $\tilde{\beta}=0.293(14)$ and $\tilde{\gamma}^{\prime}=0.52(3)$.

Measuring $\left\langle\tau_{\text {wait }}\right\rangle$ allows hence to estimate $\beta$ and to gauge whether the scaling regime is attained.

For estimating the accuracy of the critical exponents, we subsampled the data to which the fit was performed. Typically, we took $10^{3}$ sets containing each six randomly chosen data points from the selected region, to which power laws where fitted. The error of the respective exponents was then taken as an estimate of the final standard deviation [29].

\section{2. $1 D$ scaling}

The numerical results for the scaling of the densities of active sites and grains are presented in Fig. 6 for the case of chains with a variable number of $L$ sites. We find 
that the scaling exponents for the density of active sites and for the density of grains are distinct, but very close, $\beta=0.278(5)$ and $\tilde{\beta}=0.293(14)$, see (3). The same observation holds for the scaling exponents of the corresponding fluctuations, which are $\gamma^{\prime}=0.54(3)$ and $\tilde{\gamma}^{\prime}=0.52(3)$. Our resolution allows for identical scaling exponents for the density active sites and grains, a result that is consistent with the observation that the density of active sites and the mean energy per site show similar scaling behavior for a twoparameter non-conserving sandpile model [20].

In Table 1 the critical exponents obtained for the 1D autonomously adapting sandpile model are listed together with the estimates for the Manna model and directed percolation (DP). The results indicate that the universality class of the AAS model is DP, in agreement with the conjecture that generic sandpile models have directed percolation exponents [30].

The range of $p$ included in Fig. 6 reaches $p=1$ at the upper end for the probability to distribute three and not two grains. Deviations from a power law start to show up at around a distance of $p-p_{c}=\Delta p \approx 10^{-2}$ from the critical point, in particular for the fluctuations $\Delta g$ of the grain density.

\subsection{D scaling}

In Fig. 7 the results for the mean density of of active sites and grains are presented together with their respective fluctuations. For a comparison we have included data for distribution parameters $p$ that extend up to $p=1$. The fluctuations, $\Delta \rho_{a}$ and $\Delta g$ start to deviate from a power-law scaling already for $p-p_{c}$ substantially below $10^{-2}$, which is in particular evident for $\Delta g$. This pronounced deviation from a power-law scaling, which may be due to the presence of the metastable state, as discussed in Sect. 3.6, prevented us to obtain an reliable estimate for the scaling of $\Delta g$. An error bar for the respective exponent could likewise not be evaluated. The differences found between $\gamma^{\prime}$

Table 2. Two-dimensional critical exponents. The scaling exponents $\tilde{\beta}, \tilde{\gamma}^{\prime}$ and $\tilde{\sigma}$ for the grain density are defined only for the autonomously adapting sandpile model (AAS), see (3) and (5) and (7). Manna and directed percolation (DP) exponents are from [27]. For the definition of $\epsilon_{\|} / \nu_{\|}$see (9). The poor scaling behavior of the numerical data prevented us from obtaining reliable estimates for the accuracy of $\tilde{\gamma}^{\prime}$ and $\tilde{\sigma}$.

\begin{tabular}{llll}
$2 \mathrm{D}$ & $\mathrm{AAS}$ & Manna & $\mathrm{DP}$ \\
\hline$\beta$ & $0.599(9)$ & $0.639(9)$ & $0.5834(30)$ \\
$\tilde{\beta}$ & $0.632(9)$ & - & - \\
$\gamma^{\prime}$ & $0.278(12)$ & $0.367(19)$ & $0.2998(162)$ \\
$\tilde{\gamma}^{\prime}$ & 0.2 & - & - \\
$\sigma$ & $1.99(4)$ & $2.229(32)$ & $2.1782(171)$ \\
$\tilde{\sigma}$ & 1.88 & - & - \\
\hline$\epsilon_{\|} / \nu_{\|}$ & $1.51(11)$ & - & $1.549(3)$
\end{tabular}



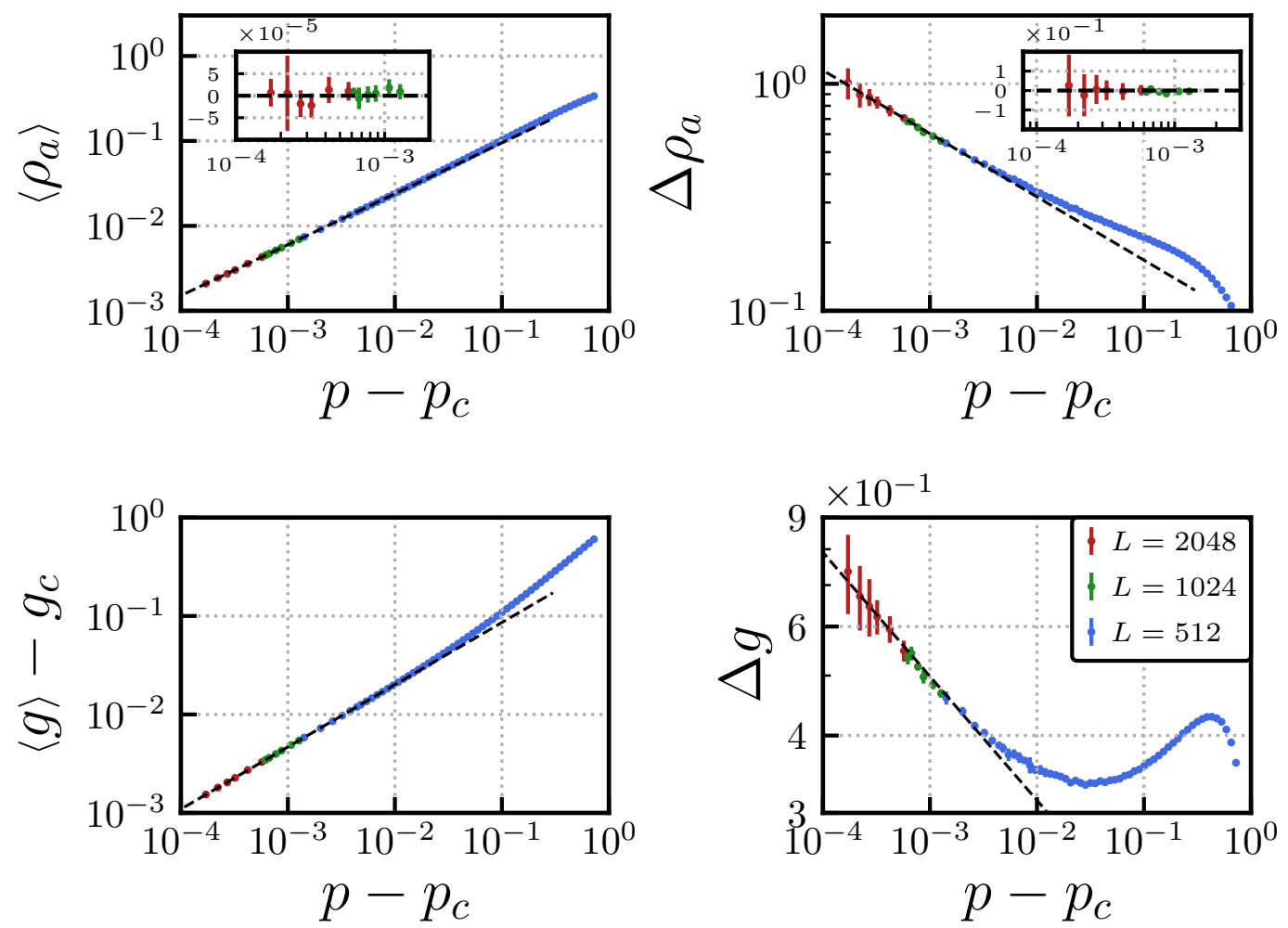

Figure 7. For two dimensional $L \times L$ square lattices, the scaling behavior as a function of the probability $p$ for toppling three and not two sites, with the data reaching to $p=1$. Dashed lines are power-law fits. Top left: The density of active sites $\rho_{a}$ fitted by $\rho_{a} \sim\left(p-p_{c}\right)^{\beta}$, with $p_{c}=0.27523(1)$ and $\beta=0.599(9)$. The inset shows the error relative to the fit, in units of $10^{-5}$. Top right: The fluctuations $\Delta \rho_{a}$ of active sites, with an exponent $\gamma^{\prime}=0.278(12)$. Bottom left: The relative density of grains $\langle g\rangle-g_{c}$ with an exponent $\tilde{\beta}=0.632(9)$ and $g_{c}=0.64686(4)$. The inset shows the error relative to the fit, in units of $10^{-1}$. Bottom right: The fluctuations $\Delta g$ of the density of grains with an exponent $\tilde{\gamma}^{\prime}=0.2$. The quality of the data prevented us to obtain reliable estimates for the accuracy of $\tilde{\gamma}^{\prime}$.

and $\tilde{\gamma}^{\prime}$, as evident from Table 2, may consequently be due to the difficulty to obtain reliable estimates in particular for $\tilde{\gamma}^{\prime}$. Regarding $\beta$ and $\tilde{\beta}$, our results indicate that they may be distinct in two dimensions, but not in $1 \mathrm{D}$, see Table 1. At this point we cannot settle definitively whether $\beta$ and $\tilde{\beta}$ are identical or not.

For the Manna model the grain density $g^{\text {Manna }}$ is the control parameter, and not a self-consistently determined quantity. All scaling relations are evaluated hence as a function of $g^{\text {Manna }}-g_{c}^{\text {Manna }}$, where $g_{c}^{\text {Manna }}$ is the critical grain density, estimated to be 0.68333(3) [27], or 0.68354(1) [23]. These estimates for the two-dimensional Manna model differ in any case from the 2D-AAS value $g_{c}=0.64686(4)$.

The scaling of $\left\langle\rho_{a}\right\rangle$ as a function of $g^{\text {Manna }}-g_{c}^{\text {Manna }}$ defines for the Manna model the order-parameter exponent $\beta$. For the two-dimensional Manna model $\beta$ has been estimated to be 0.639(9) [27] and 0.634(3) [23], with both values being compatible with 

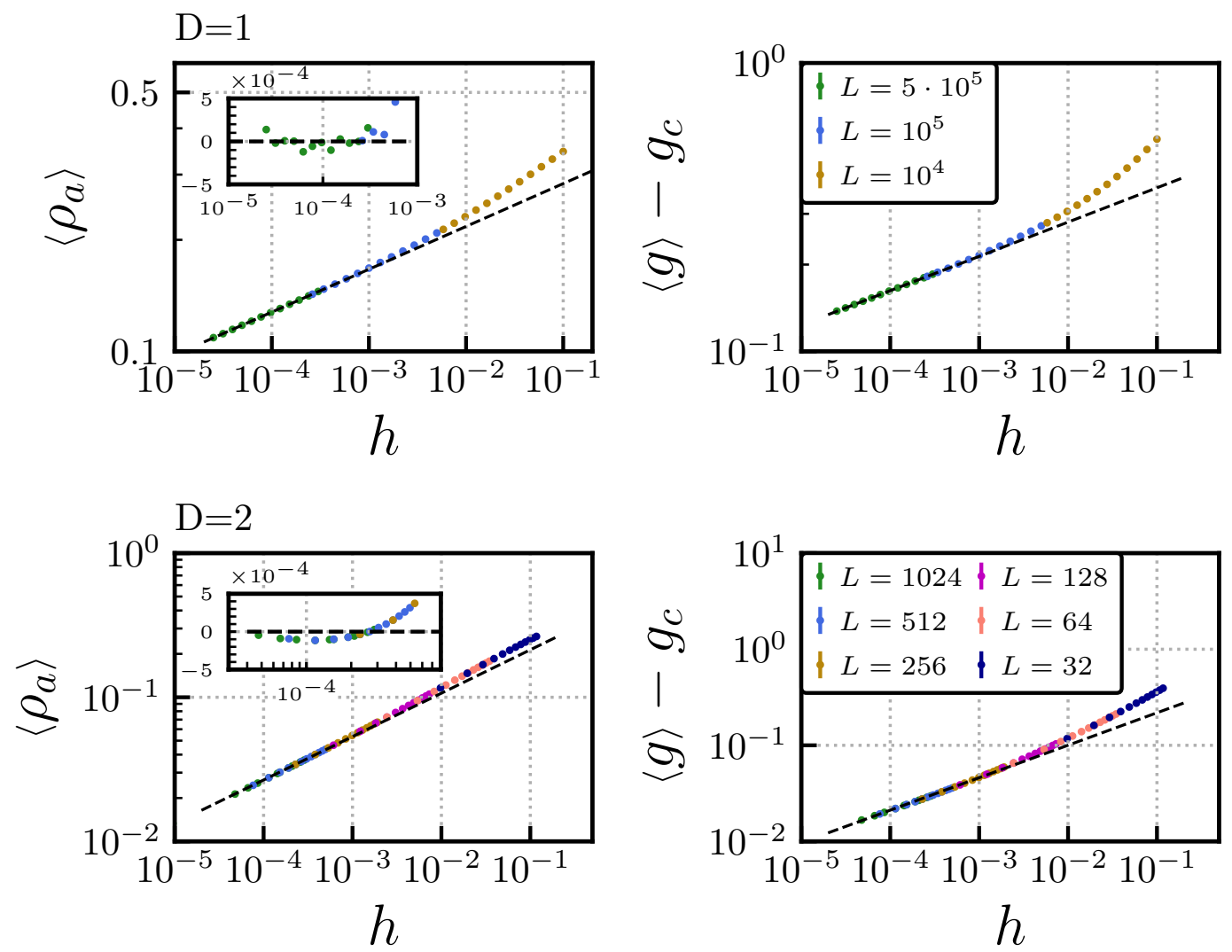

Figure 8. The density of active sites and grains as a function of the external field $h$ and for $p \approx p_{c}$. The insets show the deviation from the respective power-law fits, in units of $10^{-4}$. Upper panels: In $1 \mathrm{D}$, for chains of length $L$. The scaling exponents are $\beta / \sigma=0.116(3)$ and $\tilde{\beta} / \tilde{\sigma}=0.119(4)$ (dashed lines). Compare Sect. 3.4 and (7). Lower panels: In $2 \mathrm{D}$, for $L \times L$ lattices. The power laws for the order parameter and the grain density have exponent $\beta / \sigma=0.301(3)$ and $\tilde{\beta} / \tilde{\sigma}=0.338(12)$.

$\tilde{\beta}=0.632(9)$, as defined by (3). Our value for $\beta=0.599(9)$ is on the other side compatible with the DP estimate 0.5834(30) [23].

\subsection{External field}

There are several ways to define an external field $h$ for sandpile models. For conserving models in $D$ dimensions one usually transfers $L^{D} h$ grains between sites, which then results in the creation of active sites [31]. For directed percolation models one generates directly active sites [32].

The number of grains is not conserved for the autonomously adapting sandpile model, a circumstance that allows a straightforward definition of a field that is conjugated to the order parameter. For this purpose $L^{D} h$ grains are added randomly onto the active sublattice, where $h$ is the field strength. This is done at the start of each time step, viz of every sweep. The density of active sites and grains then scale as

$$
\left\langle\rho_{a}\right\rangle \sim h^{\beta / \sigma}, \quad\langle g\rangle-g_{c} \sim h^{\tilde{\beta} / \tilde{\sigma}}
$$



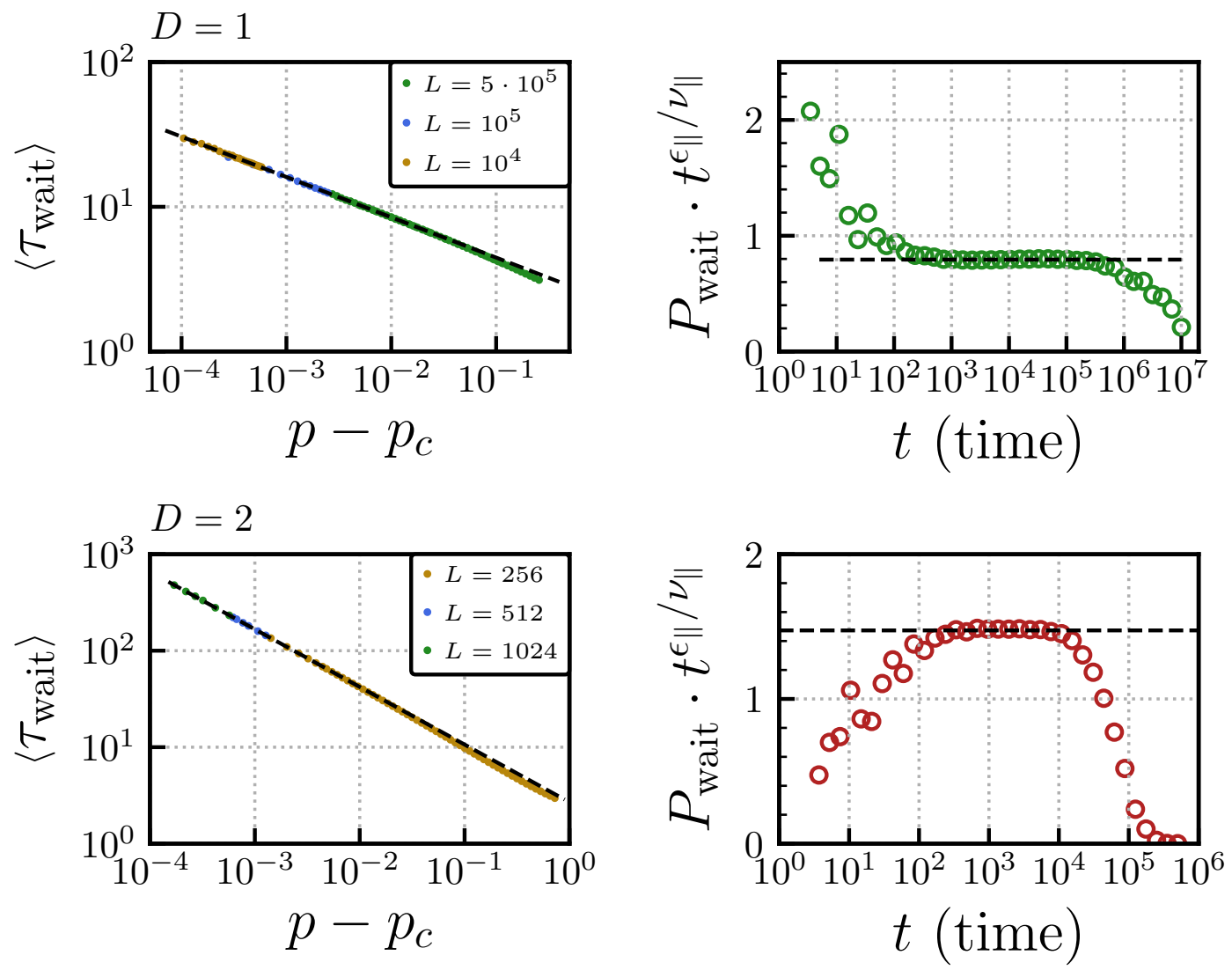

Figure 9. The waiting time and its distribution in 1D (upper panels) and in 2D (lower panels). Left panel: The mean waiting time plotted against the distance $p-p_{c}$ from the critical point. A regression analysis yields $-0.277(10)$ and $-0.597(10)$ for the exponent of the mean waiting time in $1 \mathrm{D}$ and $2 \mathrm{D}$, which agrees with the respective values of the order-parameter exponent $\beta$, see the Tables 1 and 2. Right panels: For even times, the rescaled waiting time distribution in log-log representation. The data is for $p-p_{c}=1.05 \cdot 10^{-4}$ and $L=5 \cdot 10^{5}$ in $1 \mathrm{D}$ and for $p-p_{c}=1.7 \cdot 10^{-4}$ and $L=2048$ in $2 \mathrm{D}$. A regression analysis (dashed lines) yields an exponent $\epsilon_{\|} / \nu_{\|}$, as defined by (9), of $1.86(9) / 1.51(11)$ in $1 \mathrm{D} / 2 \mathrm{D}$.

at criticality. An external field corresponding to an additional flux of grains arises naturally within the neural network interpretation of the AAS model. The field is then equivalent to the external input of an otherwise recurrent network. Formally we defined in (7) exponents $\sigma$ and $\tilde{\sigma}$, which characterize both the scaling of the field. One hence expects that $\sigma=\tilde{\sigma}$.

In Fig. 8 we present the results for both 1D and 2D systems. Shown are the field dependencies of $\left\langle\rho_{a}\right\rangle$ and $\langle g\rangle-g_{c}$ close to criticality, viz for $p=p_{c}$, where $p_{c}$ is the numerically determined critical probability to topple three and not two grains. The resulting scaling exponents $\sigma$ and $\tilde{\sigma}$ are somewhat close to the DP results, as listed in the Tables 1 and 2 . The difficulty to determine $p_{c}$ with higher accuracy may contribute to the remaining difference. 


\subsection{Waiting-time distribution}

The waiting-time distribution, which is also known as the temporal empty-interval distribution [21], is assumed to scale according to

$$
P_{\text {wait }}(t ; \delta \rho) \simeq \lambda^{-\epsilon_{\|}} \tilde{P}_{\text {wait }}\left(\lambda^{-\nu_{\|}} t ; \lambda \delta \rho\right),
$$

which implies that

$$
P_{\text {wait }}(t ; 0) \sim t^{-\epsilon_{\|} / \nu_{\|}}, \quad \epsilon_{\|} / \nu_{\|} \in(1,2) .
$$

In order for $P_{\text {wait }}=P_{\text {wait }}(t ; 0)$ to be normalizable one has that $\epsilon_{\|} / \nu_{\|}>1$. A diverging expectation value $\left\langle\tau_{\text {wait }}\right\rangle \rightarrow \infty$ at criticality needs conversely that $\epsilon_{\|} / \nu_{\|}<2$. We note that one could also use the scaling relation $\epsilon_{\|}=2 \nu_{\|}-\beta$.

In Fig. 9 the results for the mean waiting time $\left\langle\tau_{\text {wait }}\right\rangle$ and for the waiting-time distribution $P_{\text {wait }}$ are presented for both $D=1$ and $D=2$. One confirms that $\left\langle\tau_{\text {wait }}\right\rangle$ scales inversely to the density of active sites as a function of $p-p_{c}$, the distance to criticality. The waiting-time distribution $P_{\text {wait }}$ obeys (9) over several orders of magnitude, with the range being somewhat restricted in $D=2$ due to finite size effects. Overall one finds, as listed in in the Tables 1 and 2, good agreement with directed percolation.

Experimentally, the waiting-time distribution is often directly accessible, e.g. when measuring neural activity in terms of individual spikes. Alternatively one may subdivide the experimental timeline into a a series of time slices and define neural avalanches by counting the number of spikes observed in individual time slices [6, 33]. Obtaining neuronal avalanches in this way is however plagued by subsampling and other issues [7], which do not affect local quantities such as the waiting-time distribution. Observables, as the waiting-time distribution, that do not require to define neuronal avalanches in first place can hence be regarded as avalanche-free probes to absorbing phase transitions.

\subsection{The influence of metastable states}

The AB-sublattice symmetry present in bipartite models is generically broken for synchronous sandpile models when nearest-neighbor toppling rules are applied, as discussed in Sect. 2. The process of stochastically breaking the AB-symmetry may be fast or slow, as illustrated in Figs. 3 and 4 respectively for the Manna- and for the AAS model. In particular in $D=2$ we have the situation that the AB-symmetric state is transiently stable even close to criticality, see Fig. 2.

The situation is non-trivial, as the state for which all active sites are alternatingly on the $\mathrm{A}$ and the $\mathrm{B}$ sublattice, the asymmetric state, is not dynamically stable on short time scales. A small perturbation in the form of a small addition of active sites to the empty sublattice will quickly increase in magnitude, until an equilibrated but fluctuating state is reached, as shown in Fig. 4. The sublattice breaking state is then recovered once a fluctuation in the sublattice density of active sites is large enough to empty one of the two sublattices altogether, which happens however only for prolonged times. This observation suggests that the AB-sublattice symmetry is broken stochastically and not, as we also show in Sect. 4.1, by a driving molecular field. 
The presence of metastable states is not expected to alter the critical exponents, it may however affect, as we speculate here, the width of the scaling regime, with the reason being that the state for which scaling is evaluated, the symmetry-broken state, is unstable for short times. This presumption could explain the non-monotonic behavior of $\Delta g$ in Fig. 7 and that the deviations from a pure power-law scaling start in two dimensions at smaller $p-p_{c}$ than for $D=1$, compare Fig. 6 and 7 .

\section{Mean-field theory}

The evolution of the densities $\rho_{0}, \rho_{1}$ and $\rho_{a}$ of empty, singly-occupied and active sites is governed on a molecular-field level by the master equation

$$
\begin{aligned}
& \left.\rho_{0}\right|_{t+1}=\left(1-\rho_{1}\right) q_{0} \\
& \left.\rho_{1}\right|_{t+1}=\rho_{1} q_{0}+\left(1-\rho_{1}\right) q_{1},
\end{aligned}
$$

where $q_{0}$ and $q_{1}$ denote the probability to receive zero or one sandcorns from nearestneighbor toppling sites. We have used here that synchronous updating implies that a site is empty before receiving grains from other sites either because it was empty to start with, or because it was active. As described by (10), a site will be empty if it was not singly-occupied, the factor $1-\rho_{1}$, and if it did not receive grains, the factor $q_{0}$. Note that (10) and (11) are not explicitly dependent on the density $\rho_{a}=1-\rho_{0}-\rho_{1}$ of active sites. Once equilibrated, one has for all $q_{0}, q_{1} \in[0,1]$

$$
\rho_{0}=\frac{\left(1-q_{0}\right) q_{0}}{1-q_{0}+q_{1}}, \quad \rho_{1}=\frac{q_{1}}{1-q_{0}+q_{1}}, \quad \rho_{a}=\frac{\left(1-q_{0}\right)^{2}}{1-q_{0}+q_{1}} .
$$

For the evaluation of $q_{0}$ and $q_{1}$ for a $D$-dimensional hypercubic lattice we define with

$$
\tilde{q}_{0}=\left(1-\frac{1}{2 D}\right)^{2}(1-p)+\left(1-\frac{1}{2 D}\right)^{3} p
$$

the probability that a selected neighboring site of a toppling site receives zero grains. In analogy we have

$$
\tilde{q}_{1}=\frac{1}{2 D}\left[2\left(1-\frac{1}{2 D}\right)(1-p)+3\left(1-\frac{1}{2 D}\right)^{2} p\right]
$$

that a selected neighboring site of a toppling sites receives exactly one grain. One then has

$$
q_{0}=\left(1-\rho_{a}+\rho_{a} \tilde{q}_{0}\right)^{2 D}, \quad q_{1}=2 D \rho_{a} \tilde{q}_{1}\left(1-\rho_{a}+\rho_{a} \tilde{q}_{0}\right)^{2 D-1},
$$

which one needs to substitute into (12). Expanding the result in $\rho_{a}$ one finds

$$
p_{c}=\left\{\begin{array}{ll}
0.3246 & (D=1) \\
0.1481 & (D=2)
\end{array}, \quad \lim _{D \rightarrow \infty} p_{c}=0 .\right.
$$

In infinite dimensions exactly two grains are distributed during an toppling event. The average number of grains per site is

$$
g=\rho_{1}+\rho_{a}(2+p)=\frac{q_{1}+\left(1-q_{0}\right)^{2}(2+p)}{1-q_{0}+q_{1}},
$$

where $2+p$ is the average number of grains distributed by an active site. The mean-field exponents are $\beta=\tilde{\beta}=1$ and $\sigma=\tilde{\sigma}=2$. 


\subsection{Mean-field theory for two sublattices}

Eqs. (10), (11) and (15) can be generalized to the case of two sublattices. Iterating the resulting master equations one finds that the $\mathrm{AB}$-sublattice symmetry is kept, when present, and restored when starting with asymmetric initial conditions, which implies that the critical value $p_{c}$ for toppling three and not two grains remains unaffected by the presence of two distinct sublattices and that the loss of the AB-sublattice symmetry observed numerically in Figs. 3 and 4 is the result of a stochastic process, and not due to a driving molecular field. For a generalized contact process it has been shown on the other hand that the sublattice symmetry can be broken already on a mean-field level [34]. Both states, broken and symmetric, exist in this case in specific regions in phase space that are connected by a second-order phase transition, except for a limiting case where the transition is discontinuous. This is however not the case for the AAS model.

\section{Discussion}

Previously, in Sect. 3.6, we argued that the observed deviations from power-law scaling, which are particularly prominent in two dimensions and for the scaling of the density of grains, may be induced by the presence of a metastable state. A range of alternatives is worth discussing.

\subsection{Logarithmic corrections}

Multiplicative logarithmic corrections are generically present in marginal scenarios [35], in particular at the upper critical dimension [36] and at bicritical points, namely when a transition changes from second to first order [37]. For directed percolation and for the Manna universality class, which includes the conserved lattice gas [4], the upper critical dimension is four [27, 21], which implies that the deviation from power-law scaling present in Fig. 7 should not be due to a marginal scenario.

\subsection{Two parameter scaling}

The density of active sites $\rho_{a}$ is determined for the Manna model by the average number of grains per site, which acts, being a conserved quantity, as an external control parameter. For the AAS model two quantities, $\rho_{a}$ and the grain density $g$, are determined by the external control parameter, namely $p$. We find that the grain density is coupled to $\rho_{a}$, as both $\left\langle\rho_{a}\right\rangle$ and $\langle g\rangle-g_{c}$ show critical scaling, where $g_{c}$ is the critical grain density. In such a situation it is possible that the scaling flow is determined not by one, but by two running parameters, becoming hence two-dimensional. Two-parameter scaling is not very frequent, but known to be present for polymers in $\theta$ solvents [38], for weak topological insulators [39], and for integer quantum-Hall states [40]. Our findings point towards $\gamma^{\prime}=\tilde{\gamma}^{\prime}$, which would indicate that only a single scale determines the renormalization flow. However, we cannot rule out residual differences between $\beta$ and 
$\tilde{\beta}$. The deviation from power-law scaling observed in Fig. 7, could be interpreted in this case as a crossover phenomenon.

\subsection{Conserving vs. dissipative avalanches}

There are two types of avalanches in locally conserving sandpile models on lattices with open boundary conditions. Dissipative avalanches are those reaching the boundary, where grains are lost, whereas conserving avalanches stop before reaching the boundary. Dissipative and conserving avalanches differ qualitatively with respect to their statistical properties, with dissipative avalanches showing clean power-law scaling [16]. Conserving avalanches are characterized in contrast by substantial multiplicative logarithmic corrections, which are furthermore strongly size dependent [17]. For this scenario to explain the deviation of power-law scaling present in Fig. 7, the internal avalanches of the AAS model with periodic boundary conditions would need to behave akin to the conserving avalanches of abelian sandpile models with open boundaries. The rational for this putative equivalence is however presently not evident.

\section{Conclusions}

Surprisingly complex physics is found for a new dynamical systems model, the autonomously adapting sandpile (AAS) model. While energy, respectively sand is locally non conserved, an absorbing phase transition within the directed percolation universality class is observed. For the AAS model the density of grains attains a selforganized value. Pointing out that synchronous update rules on bipartite lattices allow the AB-sublattice symmetry to be broken stochastically, we argue that the metastable sublattice-symmetric state leaves its imprint on the scaling behavior, in particular in two dimensions, where the sublattice symmetric state has a prolonged lifetime even in the vicinity to criticality. The proposed model is well suited for modeling critical brain dynamics, as it mimics the dynamics of integrate and fire neurons. Open issues regard the lifetime of the metastable AB-symmetric state and the behavior on non-bipartite lattices and graphs, respectively of update rules that do not conserve the sublattice symmetry.

\section{Acknowledgments}

We thank Peter Kopietz, Malte Henkel and Dimitrije Markovic for discussions.

\section{References}

[1] Hinrichsen H 2000 Advances in Physics 49 815-958

[2] Kockelkoren J and Chaté H 2003 Physical Review Letters 90125701

[3] Henkel M and Hinrichsen H 2004 Journal of Physics A: Mathematical and General 37 R117

[4] Rossi M, Pastor-Satorras R and Vespignani A 2000 Physical Review Letters 851803

[5] Manna S 1991 Journal of Physics A: Mathematical and General 24 L363 
[6] Beggs J M and Plenz D 2003 Journal of Neuroscience 23 11167-11177

[7] Priesemann V, Valderrama M, Wibral M and Le Van Quyen M 2013 PLoS Computational Biology 9 e1002985

[8] Chialvo D R 2004 Physica A: Statistical Mechanics and its Applications 340 756-765

[9] Marković D and Gros C 2014 Physics Reports 536 41-74

[10] Gros C and Marković D 2013 Observing scale-invariance in non-critical dynamical systems AIP Conference Proceedings vol 1510 (AIP) pp 44-53

[11] Marković D, Gros C and Schuelein A 2013 Chaos: An Interdisciplinary Journal of Nonlinear Science 23013106

[12] Markovic D and Gros C 2009 New Journal of Physics 11073002

[13] Bak P 1998 How Nature Works: The Science of Self-Organized Criticality Copernicus, New York, 1996; HJ Jensen, Self-Organized Criticality (Cambridge University Press, Cambridge)

[14] Bak P, Tang C and Wiesenfeld K 1987 Physical Review Letters 59381

[15] Levina A and Priesemann V 2017 Nature Communications 815140

[16] Drossel B 2000 Physical Review E 61 R2168

[17] Dickman R and Campelo J 2003 Physical Review E 67066111

[18] Vespignani A, Dickman R, Muñoz M A and Zapperi S 1998 Physical Review Letters 815676

[19] Gros C 2015 Complex and adaptive dynamical systems: A primer (Springer)

[20] Basu U, Basu M and Mohanty P 2013 The European Physical Journal B 86236

[21] Henkel M, Hinrichsen H, Lübeck S and Pleimling M 2008 Non-equilibrium phase transitions vol 1 (Springer)

[22] Lübeck S 2001 Physical Review E 64016123

[23] Lee S B 2014 Physical Review E 89062133

[24] Hohenberg P C and Halperin B I 1977 Reviews of Modern Physics 49435

[25] Lübeck S and Heger P 2003 Physical Review E 68056102

[26] Daga B and Ray P 2018 arXiv preprint arXiv:1812.10704

[27] Lübeck S 2004 International Journal of Modern Physics B 18 3977-4118

[28] Basu M, Basu U, Bondyopadhyay S, Mohanty P and Hinrichsen H 2012 Physical review letters 109015702

[29] Hinrichsen H 2006 Physica A: Statistical Mechanics and its Applications 3691 - 28 ISSN 0378-4371 fundamental Problems in Statistical Physics

[30] Mohanty P and Dhar D 2002 Physical Review Letters 89104303

[31] Lübeck S 2002 Physical Review E 65046150

[32] Lübeck S and Willmann R 2002 Journal of Physics A: Mathematical and General 3510205

[33] Hesse J and Gross T 2014 Frontiers in systems neuroscience 8166

[34] de Oliveira M M and Dickman R 2011 Physical Review E 84011125

[35] Wegner F J and Riedel E K 1973 Physical Review B 7248

[36] Lübeck S 1998 Physical Review E 582957

[37] Kenna R, Johnston D and Janke W 2006 Physical Review Letters 96115701

[38] Colby R H and Rubinstein M 1990 Macromolecules 23 2753-2757

[39] Mong R S, Bardarson J H and Moore J E 2012 Physical Review Letters 108076804

[40] Werner M A, Brataas A, Von Oppen F and Zaránd G 2015 Physical Review B 91125418 